\title{
Microwave drying of Siraitia grosvenorii and its effect on the Vitamin C content
}

\author{
Zhide Zhou ${ }^{\mathrm{a}}$, Guizhi Huang ${ }^{\mathrm{b}}$, Guiyin $\mathrm{Li}^{\mathrm{c} *}$ \\ School of Life and Environmental Sciences, Guilin University of Electronic Technology, Guilin, \\ Guangxi 541004, China \\ azzdlgy@163.com, ${ }^{b} 1377091950 @ q q . c o m,{ }^{c}$ liguiyin01@163.com(*Corresponding author)
}

\begin{abstract}
Keywords: Microwave drying; Vitamin C; Siraitia grosvenorii; Changes
Abstract: Siraitia grosvenorii (Luo hanguo, LHG) is a unique edible and medicinal plant in China. Drying the LHG with a rapid, highly efficient method and keeping good quality are urgent problems. In this paper, the changes of Vitamin C (VC) content in LHG under microwave drying were studied. The effects of key drying parameters (microwave power, drying time and microwave intermittent ratio) on the quality of LHG have been investigated by orthogonal experiments to reduce the loss of $\mathrm{VC}$ in the process of microwave drying. The optimum drying parameters for drying LHG were microwave power of $80 \mathrm{~W}$, drying time of $30 \mathrm{~min}$ and microwave intermittent ratio of 1: 1 . The optimally treated LHG has $34.072 \mathrm{mg} / 100 \mathrm{~g}$ of VC content. These results have indicated that the microwave-heating not only prolongs the storage duration of the LHG but also reduces the moisture content and VC content, while the natural yellow-green color of the LHG is not affected much.
\end{abstract}

\section{Introduction}

Siraitia grosvenorii, commonly known as Luo hanguo (LHG), is the first batch of medicinal and edible species in China's Ministry of Health. It has the reputation of "Oriental fruit" and "Longevity fruit" in Southeast Asia and Western countries, and it is also a traditional specialty in northern Guangxi province of China [1,2]. To date, several classes of compounds were isolated from LHG such as triterpenoids (especially mogroside), flavonoids, vitamins, amino acid, saccharides, volatile oil and so on. LHG has antitussive, anti-asthmatic, anti-oxidation, liver-protection, glucose-lowering, immune-regulation, and anti-cancer effects $[3,4]$.

LHG is a perishable fruit and its harvesting and storage time are very limited because of high moisture content, easy mildew and rot, easy discoloration and browning. Drying is a common and cheap alternative to preserve LHG against microbial and enzymatic activity, prolong the storage period, reduce postharvest losses and improve the utilization value [5]. Lately new and innovative drying methods that increase the drying rate and enhance product quality have achieved considerable attention.

Microwave drying, a relatively new technology, has been recommended as a fast and effective alternative drying technique due to low energy efficiency, simple operation and good quality of dried products[6, 7]. Oraporn Bualuang et al.[6] have shown the positive impact of microwave drying on the quality attributes of germinated and enhancing the antioxidant compounds present in this food product.

Vitamin $\mathrm{C}$ (VC), as an antioxidant present in fruits and vegetables, is a water-soluble vitamin that is necessary for normal growth and development. Its content in fruits could be reduce during the drying process due to its high temperature and moisture-sensitivity [8]. LHG fruit is rich in vitamin C. The content of vitamin C in the fresh fruit of LHG reached 339-461 mg/100 g, but it decreased to $24.6-38.7 \mathrm{mg} / 100 \mathrm{~g}$ in the dried fruit [3]. In this paper, the effect of microwave drying on the content of $\mathrm{VC}$ in LHG and the changes of VC content under different microwave drying parameters have been investigated. 


\section{Experimental}

\section{Chemicals and apparatus}

Metaphosphoric acid, oxalic acid and methanol was purchased from Sinopharm Chemical Reagent Co., Ltd (Tianjin, China). LHG fruit was harmonious provided by GuilinSanbao pharmaceutical Co., Ltd (Guangxi, China). VC standard reagent was purchased from Tianjin Fuchen chemical reagent Co., Ltd (Tianjin, China). All other reagents were of analytical grade and used without any further purification. Millipore uitrapure water with specific $18 \mathrm{M} \Omega \mathrm{cm}$ was used throughout the experiments.

The microwave drying was conducted in a microwave oven (Midea, EG823LC8-NS, China). High performance liquid chromatography(HPLC) equipment was Water HPLC 515 (Waters, Milford, MA).

\section{Preparation of VC standard solution and sample solution}

VC standard stock solutions: VC standard stock solutions $(1.0 \mathrm{mg} / \mathrm{mL})$ were prepared fresh daily, $50.0 \mathrm{mg}$ dried VC standard reagent was accurately weighed and dissolved in $0.05 \%$ metaphosphoric acid solution.

Sample solution: A sample of dried LHG fruits was weighed and blended at high speed in 100 $\mathrm{mL}$ of $5 \mathrm{mg} / \mathrm{mL}$ oxalic acid solution ( $4 \square$ ), homogenate after leaching for $15 \mathrm{~min}$, and filtered. The filtered solution was centrifugal at $4000 \mathrm{rpm}$ for $2 \mathrm{mins}$ at $4000 \mathrm{rpm}$ and filtered under pressure vacuum filtration. Then, the filtered solution was diluted with $5 \mathrm{mg} / \mathrm{mL}$ oxalic acid solution to total of $250 \mathrm{~mL}$. Preparations of the sample solutions were performed just before the HPLC determination.

\section{Optimization study for microwave treatment}

The experimental design for this study was divided into two major parts. Firstly, single factor experiments were performed to determine the appropriate range of microwave drying conditions. Three key factors influencing the content of VC in LHG were investigated, including microwave power (A), drying time (B) and microwave intermittent ratio (C). Secondly, A three factors and three levels of orthogonal experiment $\left(\mathrm{L}_{9}\left(3^{3}\right)\right)$ was employed to find out the optimum microwave drying conditions based on single factor experiments. The microwave power level was $80 \mathrm{~W}, 240 \mathrm{~W}$ and $400 \mathrm{~W}$. The drying time level was $100 \mathrm{~min}, 30 \mathrm{~min}$ and $20 \mathrm{~min}$, and microwave intermittent ratio is 0 , $1: 1$ and 2:1. The orthogonal experiment design at the given range of the above parameters in terms of actual values is presented in Table 1. Software of orthogonal designing assistant II V3.1 was used for the evaluation of the statistical experimental design.

\section{HPLC analysis the content of VC}

The content of VC was analyzed by HPLC according to the method previously described, with slight modification [9]. The sample solutions was filtrated through a $0.45 \mu \mathrm{m}$ filter, A C18 analytical column $(4.6 \mathrm{~mm} \times 250 \mathrm{~mm})$ was used for the separation at a temperature of $25^{\circ} \mathrm{C}$. The mobile phase was $0.05 \%$ phosphoric acid - methanol solution (98:2) solution. The flow rate was $1.0 \mathrm{~mL} / \mathrm{min}$ for a total run time of $20 \mathrm{~min}$. The injection volume was $10 \mu \mathrm{L}$ for all samples. The detector was set at 242 $\mathrm{nm}$ for simultaneous monitoring of the different samples. For quantification, calibration curves of external standard solutions of $\mathrm{VC}$ were used. Extraction was carried out in duplicate and chromatographic analyses for each extract were determined in triplicate.

\section{Moisture content determination}

The moisture content was analyzed according to GB / T5009.3 - 2003 method ("Determination of moisture content in food") in each stage of microwave drying process and caculated as the following equation.

Moisture Content $=($ Weight before drying - Weight after drying $) /($ Weight before drying $) \times 100 \%$

\section{Results and Discussion}

\section{HPLC analysis the content of VC}

Vitamin $\mathrm{C}$ content in fruits would reduce during the drying process because of its high 
temperature and moisture-sensitivity [10]. Several analytical methods have been reported for the determination of vitamin $\mathrm{C}$ using titrimetry, spectrometry and amperometry [8]. In the study, HPLC was used to analysis the content of VC in the process of microwave drying. Fig. 1 was the HPLC chromatograms of the VC standard solution and extractive solution of dried LHG fruits. Seen from Fig. 1, the retention time of VC standard solution was $2.475 \mathrm{~min}$, and $2.519 \mathrm{~min}$ in dried LHG. VC standard reagent was used as the standard, a linear correlation between peak areas and VC concentration was obtained. The obtained linear regression equation was $\mathrm{Y}=28961873.4204 \mathrm{X}-1222195.5007 \quad\left(\mathrm{R}^{2}=0.9959\right)$, suggesting that $\mathrm{VC}$ standard concentration presented a good linear relation with areas in the range of 0.08 to $0.5 \mathrm{mg} / \mathrm{mL}$.
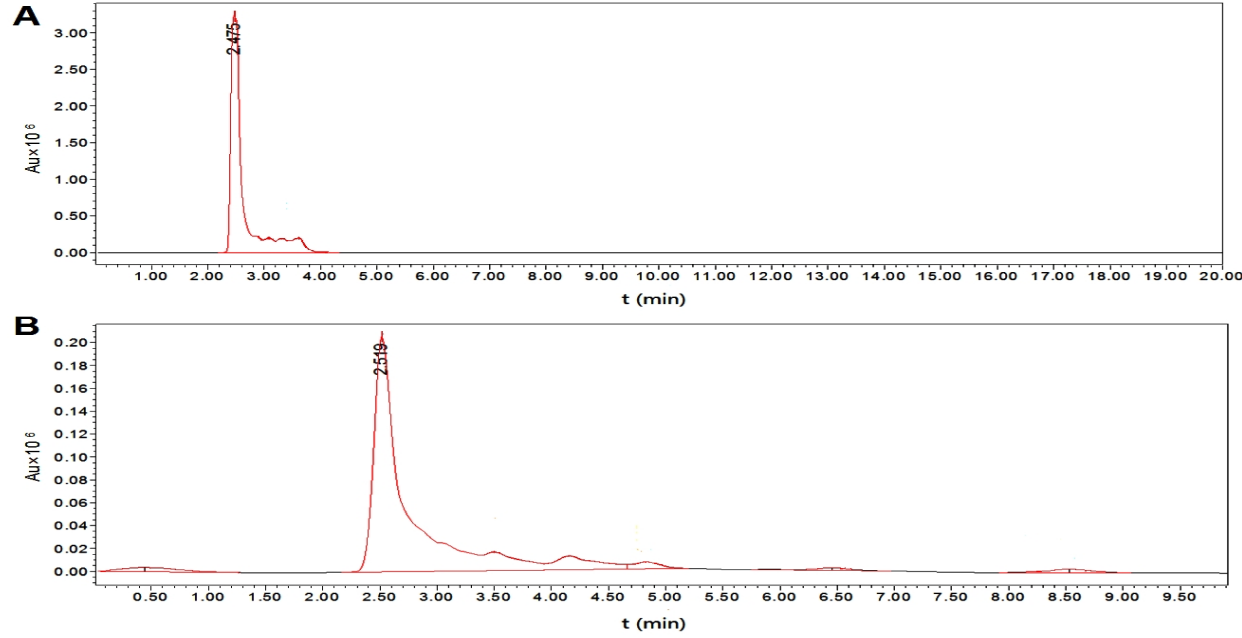

Fig. 1 HPLC chromatograms of the VC standard solution (A) and extractive solution of dried LHG fruits (B)

\section{Optimization study for microwave treatment by orthogonal experiment}

In this study, integrating the results from the single factor experiments, three key factors including including microwave power (A), drying time (B) and microwave intermittent ratio (C) influencing the content of VC were selected. Subsequently, a three-factor and three-level orthogonal experiment was designed according to the $\mathrm{L}_{9}\left(3^{3}\right)$ table. The results were shown in Table 1.

Table 1 Three-factor and three-level and the results of orthogonal experimental

\begin{tabular}{lllll}
\hline Fumber & $\begin{array}{l}\text { A: Microwave } \\
\text { power }(\mathrm{W})\end{array}$ & $\begin{array}{l}\text { B: Drying time } \\
(\mathrm{min})\end{array}$ & $\begin{array}{l}\mathrm{C} \text { : Microwave } \\
\text { intermittent ratio }\end{array}$ & $\begin{array}{l}\text { VC } \\
(\mathrm{mg} / 100 \mathrm{~g})\end{array}$ \\
\hline 1 & 80 & 100 & 0 & 26.173 \\
2 & 80 & 30 & $1: 1$ & 33.836 \\
3 & 80 & 20 & $2: 1$ & 28.474 \\
4 & 240 & 100 & $1: 1$ & 21.336 \\
5 & 240 & 30 & $2: 1$ & 25.921 \\
6 & 240 & 20 & 0 & 26.500 \\
7 & 400 & 100 & $2: 1$ & 17.210 \\
8 & 400 & 30 & 0 & 21.505 \\
9 & 400 & 20 & $1: 1$ & \\
K1 & 29.494 & 21.573 & 24.726 & \\
K2 & 24.586 & 27.087 & 25.115 & \\
K3 & 19.629 & 25.049 & 23.868 & \\
R & 9.865 & 5.514 & 1.247 & \\
\hline
\end{tabular}

According to the value of range $\mathrm{R}$ in Table1, microwave power (factor $\mathrm{A}$ ) exerted the most significant effect on the content of $\mathrm{VC}$, and the order of importance that influenced the content of VC was found to be A (microwave power) $>\mathrm{B}$ (drying time) $>\mathrm{C}$ (microwave intermittent ratio). The optimum microwave drying conditions were A1B2C2. That was microwave power of $80 \mathrm{~W}$, drying time of $30 \mathrm{~min}$, and microwave intermittent ratio of $1: 1$. Under the optimum microwave drying conditions, the content of VC in dried LHG was $33.836 \mathrm{mg} / 100 \mathrm{~g}$. 


\section{The physicochemical properties of the dried LHG}

Three batches of experiments were carried out using the recommended optimum process parameters and the results were shown in Table 2. The mean content of VC was $34.072 \mathrm{mg} / 100 \mathrm{~g}$. The results of verifying experiments were very close to the optimization analysis values. At the same time, the moisture content of $12.55 \%$ was obtained in the dried LHG fruits. According to the Chinese Pharmacopoeia (2015), the moisture content in dried LHG fruit cannot exceed $15 \%$. Thus, the quality of Siraitia grosvenorii dried by microwave was in accordance with the national requirements.

Fig. 2 was the pictures of fresh LHG and dried LHG treated with optimum microwave drying conditions. Seen from Fig. 2, there is a big difference between the color and shape of LHG fruits before and after drying. The appearance of fresh LHG fruits is egg shape or oval shape with light green color. After microwave drying, the dried fruit has natural shape with yellow-green or golden yellow surface, the peel and pulp were connected together, and retained faint scent of fresh LHG fruits.

Table 2 The validity results under the optimum microwave drying conditions

\begin{tabular}{llllllll}
\hline Factors & $\begin{array}{l}\text { Microwave } \\
\text { power }(\mathrm{W})\end{array}$ & $\begin{array}{l}\text { Drying } \\
\text { time } \\
\text { Number }\end{array}$ & $\begin{array}{l}\text { Microwave } \\
\text { intermittent } \\
\text { ratio }\end{array}$ & $\begin{array}{l}\text { VC } \\
\text { content } \\
(\mathrm{mg} / 100 \mathrm{~g})\end{array}$ & $\begin{array}{l}\text { Mean VC } \\
\text { content } \\
(\mathrm{mg} / 100 \mathrm{~g})\end{array}$ & $\begin{array}{l}\text { Moisture } \\
\text { content } \\
(\%)\end{array}$ & $\begin{array}{l}\text { Mean } \\
\text { moisture } \\
\text { content }(\%)\end{array}$ \\
\hline 1 & 80 & 30 & $1: 1$ & 34.038 & & 12.66 & \\
2 & 80 & 30 & $1: 1$ & 33.895 & 34.072 & 12.62 & 12.55 \\
3 & 80 & 30 & $1: 1$ & 34.282 & & 12.38 & \\
\hline
\end{tabular}
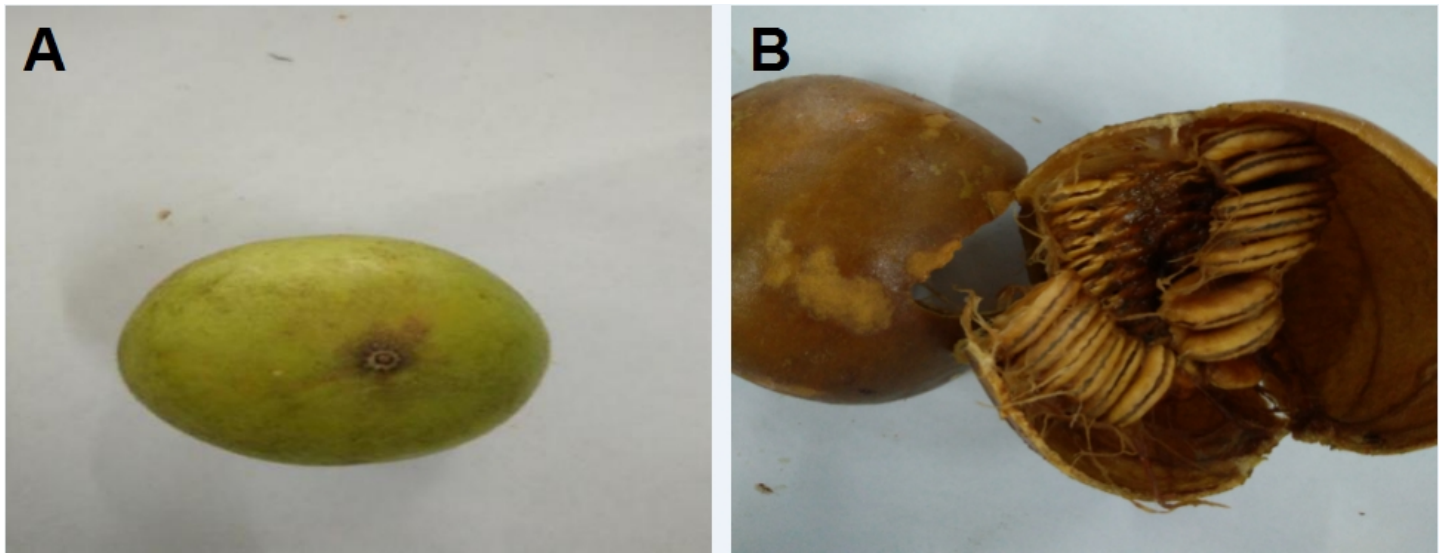

Fig. 2 Pictures of fresh LHG (A) and dried LHG treated with microwave drying (B)

\section{Conclusions}

Vitamin C content in LHG would be reduce during the drying process due to its high temperature and moisture-sensitivity, which would affect seriously the the quality of dried LHG. In this paper, the changes of VC content in LHG under different microwave drying conditions were investigated. The optimum drying parameters was microwave power of $80 \mathrm{~W}$, drying time of $30 \mathrm{~min}$, and microwave intermittent ratio of 1: 1 . Under the optimum microwave drying conditions, the content of $\mathrm{VC}$ in dried LHG $34.072 \mathrm{mg} / 100 \mathrm{~g}$, the moisture content was $12.55 \%$, and retained faint scent of fresh LHG fruits with yellow-green or golden yellow surface. This study provides a novel and environmentally safe drying technique and increase the nutritive quality of LHG.

\section{Acknowledgements}

This work was supported by the National Science Foundation of Guangxi province of China (Nos. 2016GXNSFAA380011 and 2016GXNSFAA380080). 


\section{References}

[1] C. Fang, Q. Wang, X. Liu, and G. Xu, J. Pharm. Biomed. Anal., Vol. 145. (2017), pp. 158.

[2] Z. Luo, H. Shi, K. Zhang, X. Qin, Y. Guo, and X. Ma, J. Sep. Sci., Vol. 39. (2016), pp. 4124.

[3] C. Li, L.M. Lin, F. Sui, Z.M. Wang, H.R. Huo, L. Dai, and T.L. Jiang, Chin. J. Nat. Medicines, Vol. 12. (2014), pp. 89.

[4] Z. X. Qing, H. Zhao, Q. Tang, C. M. Mo, P. Huang, P. Cheng, P. Yang, X. Y. Yang, X. B. Liu, Y. J. Zheng, and J. G. Zeng, J. Pharm. Biomed. Anal., Vol. 138. (2017), pp. 240.

[5] R. Pande, H. N. Mishra, and M. N. Singh, J. Agric. Food Chem., Vol. 60. (2012), pp. 3809.

[6] O. Bualuang, D. I. Onwude, and K. Pracha, J. Sci. Food Agric., Vol. 97. (2017), pp. 2999.

[7] E. Horuz, H. Bozkurt, H. Karatas, and M. Maskan, Food Chem., Vol. 230. (2017), pp. 295.

[8] G. Dadali, and B. Ozbek, Int. J. Food Sci. Nutr., Vol. 60. (2009), pp. 21.

[9] F. Gu, F. Huang, G. Wu, and H. Zhu, Molecules, Vol. 23. (2018), pp. 370.

[10] J. Yi, B. Kebede, K. Kristiani, T. Grauwet, A. Van Loey, and M. Hendrickx, Food Chem., Vol. 249. (2018), pp. 202. 